\title{
A FPC-ROOT Algorithm for 2D-DOA Estimation in Sparse Array
}

\author{
Wenhao Zeng, ${ }^{1}$ Hongtao Li, ${ }^{1}$ Xiaohua Zhu, ${ }^{1}$ and Chaoyu Wang ${ }^{2}$ \\ ${ }^{1}$ Nanjing University of Science and Technology, Nanjing, Jiangsu 210094, China \\ ${ }^{2}$ China Shipbuilding 724 Research Institutions, Nanjing, Jiangsu 210003, China \\ Correspondence should be addressed to Hongtao Li; liht@njust.edu.cn
}

Received 24 December 2015; Revised 11 March 2016; Accepted 22 March 2016

Academic Editor: Jeich Mar

Copyright (C) 2016 Wenhao Zeng et al. This is an open access article distributed under the Creative Commons Attribution License, which permits unrestricted use, distribution, and reproduction in any medium, provided the original work is properly cited.

\begin{abstract}
To improve the performance of two-dimensional direction-of-arrival (2D DOA) estimation in sparse array, this paper presents a Fixed Point Continuation Polynomial Roots (FPC-ROOT) algorithm. Firstly, a signal model for DOA estimation is established based on matrix completion and it can be proved that the proposed model meets Null Space Property (NSP). Secondly, left and right singular vectors of received signals matrix are achieved using the matrix completion algorithm. Finally, 2D DOA estimation can be acquired through solving the polynomial roots. The proposed algorithm can achieve high accuracy of 2D DOA estimation in sparse array, without solving autocorrelation matrix of received signals and scanning of two-dimensional spectral peak. Besides, it decreases the number of antennas and lowers computational complexity and meanwhile avoids the angle ambiguity problem. Computer simulations demonstrate that the proposed FPC-ROOT algorithm can obtain the 2D DOA estimation precisely in sparse array.
\end{abstract}

\section{Introduction}

Two-dimensional direction-of-arrival (2D DOA) [1-4] estimation plays an important role in array signal processing, and it usually utilizes L-shaped arrays, planar arrays, vector sensors, and so forth, to estimate the two-dimensional target angle. Compared with 2D DOA estimation of other arrays [5, 6], 2D DOA estimation of planar array is asymptotically unbiased where the target position can be determined precisely without the problem of angle ambiguity. Moreover, the phased-array radar in the planar array can utilize waveform diversity which makes it suitable for multiple targets and complex environments with enhanced performance of target recognition and target tracking. However, the planar array requires a large amount of array elements which results in tremendous demands of hardware equipment and increases the complexity and cost of design. Comparatively, the sparse array needs only a quite smaller number of elements, so it is a feasible remedy for the abovementioned problems, whereas, in this case, the problem of substantially rising average side lobes occurs, which accounts for its uncommon applications so far. Paper [5] lowers computational complexity of DOA estimation by introducing a preprocessing transformation matrix but has poor performance in sparse array. Paper [7] achieves high-resolution DOA estimation in sparse array; however, it only works in uniform linear array (ULA).

Matrix completion [8-10] is an extension of compressive sensing (CS) $[11,12]$ and has been widely applied to image processing, remote sensing, and many other engineering fields $[13,14]$. On the basis of low-rank matrix, matrix completion can recover the full matrix from an incomplete set of matrix entries with high probability by solving nuclearnorm optimization. Paper [15] proposed a fixed point and Bregman iterative methods for matrix rank minimization, which is a very fast, robust, and powerful algorithm. Paper [16] introduces a particularly simple yet highly efficient alternating projection algorithm and this algorithm is able to recover matrix from the minimum number of measurements necessary. Paper [17] provides a necessary and sufficient condition that quantifies when this heuristic successfully finds the minimum rank solution of a linear constraint set. The sparse received signal can be recovered to the full received signal by matrix completion, so that matrix completion has been applied to DOA estimation $[14,18]$.

This study proposes a Fixed Point Continuation Polynomial Roots (FPC-ROOT) algorithm. The proposed algorithm 


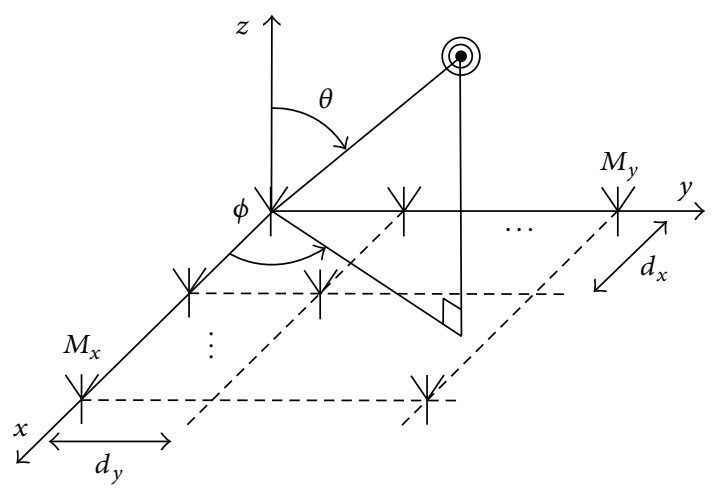

Figure 1: The uniform rectangular array.

establishes a signal model of matrix completion for 2D DOA estimation based on low-rank property of target in twodimensional space domain, which turns out to meet the Null Space Property (NSP) and ensure the feasibility of 2D DOA estimation via matrix completion. By contrast to conventional algorithms, the proposed algorithm obtains left and right singular vectors of received signal matrix directly from the output of matrix completion algorithm instead of eigendecomposing autocorrelation matrix of received signal, for the benefit of lower dimensions. Besides, the proposed algorithm can avoid scanning of the 2D spectral peak by solving the polynomial roots, which reduces the computational complexity. In addition, it can estimate target angle accurately with less number of array units by adopting matrix completion.

\section{Signal Model in Sparse Array}

2.1. The Signal Model of $2 D$ DOA Estimation. Suppose that the uniform rectangular array (URA) [3] is depicted in Figure 1. $M_{x}$ and $M_{y}$ are the number of elements in $x$-direction and $y$ direction, respectively. The corresponding element spacing is $d_{x}$ and $d_{y}$.

Assume that there are multiple targets in the space. The number of snapshots is $W$ and the number of targets is $k$, where the waveform of the $i$ th $(i=1,2, \ldots, k)$ target is $s_{i}(t)$ and $t=1,2, \ldots, W$. The target angles are $\left(\phi_{i}, \theta_{i}\right)$, where $\phi_{i}$ and $\theta_{i}$ express the elevation and azimuth angles of the $i$ th target, respectively. Suppose the uncorrelated narrow-band signals are received in far field [19]; thus, the received signal of $M_{x}$ elements in $x$-direction can be written as

$$
L_{x}(t)=A_{x} S(t)+N_{x}(t)
$$

where $S(t)=\operatorname{diag}\left(s_{i}(t)\right)$ is a diagonal matrix of $k \times k$ dimensions, the first $k$ elements of $s_{i}(t)$ are nonzero which correspond to $k$ targets, and $N_{x}(t)$ is the noise vector of the array. $A_{x}$ is a $M_{x} \times k$ dimensional steering vector, which can be given by

$$
A_{x}=\left[a_{x}\left(\phi_{1}, \theta_{1}\right), a_{x}\left(\phi_{2}, \theta_{2}\right), \ldots, a_{x}\left(\phi_{k}, \theta_{k}\right)\right]
$$

Assume the wavelength of received signal is $\lambda$; then, $a_{x}\left(\phi_{i}, \theta_{i}\right)$ is

$$
\begin{aligned}
{\left[a_{x}\left(\phi_{i}, \theta_{i}\right)\right]_{m_{x}}=e^{j(2 \pi / \lambda) d_{x} \sin \left(\theta_{i}\right) \cos \left(\phi_{i}\right)\left(m_{x}-1\right)}, } & \\
& m_{x}=1,2, \ldots, M_{x} .
\end{aligned}
$$

Similarly, the received signal of $M_{y}$ elements in $y$-direction can be represented as

$$
L_{y}(t)=A_{y} S(t)+N_{y}(t)
$$

where $N_{y}(t)$ is the noise vector of the array. $A_{y}$ is $M_{y} \times k$ dimensional steering vector, which can be denoted as

$$
A_{y}=\left[a_{y}\left(\phi_{1}, \theta_{1}\right), a_{y}\left(\phi_{2}, \theta_{2}\right), \ldots, a_{y}\left(\phi_{k}, \theta_{k}\right)\right] \text {, }
$$

where $a_{y}\left(\phi_{i}, \theta_{i}\right)$ is defined as

$$
\begin{array}{r}
{\left[a_{y}\left(\phi_{i}, \theta_{i}\right)\right]_{m_{y}}=e^{j(2 \pi / \lambda) d_{y} \sin \left(\theta_{i}\right) \sin \left(\phi_{i}\right)\left(m_{y}-1\right)},} \\
m_{y}=1,2, \ldots, M_{y} .
\end{array}
$$

In conclusion, the received signal model of full array is demonstrated as

$$
X(t)=A_{x} S(t) A_{y}^{T}+N_{R}(t), \quad t=1, \ldots, N .
$$

When the power of noise matrix $N_{R}(t)$ is much smaller than the signal power, we can get that $\operatorname{rank}(X(t)) \leq$ $\operatorname{rank}(S(t))=k$; in other words, matrix $X(t)$ is low-rank [10]. And matrix in (7) is consistent with strong incoherence property [15], so it can be recovered precisely with high probability via matrix completion.

2.2. Matrix Completion. With regard to the sparsity of signals, CS can sample signals at far lower sampling frequency than Nyquist sampling frequency and meanwhile reconstruct original signals precisely. In CS, the target to be recovered is a vector; however, on some practical occasions, it normally refers to a matrix and is sensitive to data missing, data corruption, and so on.

Matrix completion is an extension of CS. CS exploits the sparsity of signals; nevertheless, matrix completion utilizes the sparsity of matrix singular values, namely, the low-rank property, and reconstructs full matrix by solving nuclearnorm optimization.

Suppose $M \in R^{n \times n}$ is an original low-rank matrix, where $\operatorname{rank}(M)=r, r \ll n$. Matrix completion refers to recovering the whole elements of matrix $M$ from its partial elements. Let the available entries of matrix be $\left\{M_{i j},(i, j) \in \Omega\right\}$, where $\Omega$ is a random sampled subset of the whole elements; then, the matrix completion can be denoted as [10]

$$
\begin{array}{ll}
\min & \|X\|_{*}, \\
\text { s.t. } & X_{i j}=M_{i j}, \quad(i, j) \in \Omega,
\end{array}
$$

where matrix $X$ is the estimated value of matrix $M$; $\|X\|_{*}$ represents the nuclear norm of the matrix $X$. 
2.3. The Sparse Array Model. We sample elements from URA at random. Let the number of sampled elements be $m$ with the corresponding element positions unchanged and the unsampled units removed; thus, a new sparse array is set up. To recover matrix precisely via matrix completion, the required number of samples is $m \geq r\left(n_{1}+n_{2}-r\right)$ [10], while $n_{1}, n_{2}$ are the numbers of rows and columns of the matrix, and $r$ is the rank of the matrix. Suppose the received signal matrix in sparse array is $X_{s}(t)$; then, $X_{s}(t)$ is related to $X(t)$ by

$$
\begin{aligned}
& X_{s}(t)_{i j}=X(t)_{i j}, \quad(i, j) \in \Omega, \\
& X_{s}(t)_{i j}=0, \quad(i, j) \notin \Omega,
\end{aligned}
$$

where $\Omega$ is the set of element positions in sparse array.

\section{The Proposed Algorithm}

3.1. The Signal Model for 2D-DOA Estimation Based on Matrix Completion. According to (7), the received signal matrix $X(t)$ in URA is low-rank. Regard $X_{R}(t)$ as the recovered matrix by matrix completion, so it can be deduced that

$$
\begin{aligned}
\min & \left\|X_{R}(t)\right\|_{*} \\
\text { s.t. } & X_{R}(t)_{i j}=X_{s}(t)_{i j}, \quad(i, j) \in \Omega .
\end{aligned}
$$

If a signal model meets NSP, the rank minimization of this model is equivalent to its nuclear-norm minimization [13]. So we introduce the projection $P_{\Omega}$ as follows:

$$
P_{\Omega}(M)= \begin{cases}M_{i j}, & (i, j) \in \Omega \\ 0, & (i, j) \notin \Omega .\end{cases}
$$

From (11), we can see that (10) can be expressed as

$$
\begin{aligned}
\min & \left\|X_{R}(t)\right\|_{*}, \\
\text { s.t. } & P_{\Omega}\left(X_{R}(t)\right)=P_{\Omega}\left(X_{s}(t)\right),
\end{aligned}
$$

where $X_{R}(t)$ is the estimated matrix. The null space of projection $P_{\Omega}$ is

$$
\operatorname{Null}\left(P_{\Omega}\right)=\left\{M \in R^{n_{1} \times n_{2}}: P_{\Omega}(M)=0\right\} .
$$

According to NSP, we are unable to recover a matrix if it belongs to the null space of projection $P_{\Omega}$. From (4), we can get that

$$
\begin{array}{r}
{\left[a_{x}\left(\phi_{i}, \theta_{i}\right)\right]_{m_{x}}=e^{j(2 \pi / \lambda) d_{x} \sin \left(\theta_{i}\right) \cos \left(\phi_{i}\right)\left(m_{x}-1\right)} \neq 0,} \\
m_{x}=1,2, \ldots, M_{x} .
\end{array}
$$

In (7), the arbitrary elements of $A_{x}$ and $A_{y}$ are both nonzero and the first $k$ elements in $s_{i}(t)$ are nonzero, so the diagonal elements of $k \times k$ dimensional diagonal matrix are nonzero. Arbitrary elements of $X(t)$ are nonzero based on matrix multiplication property. Therefore, it can be concluded that whichever sampling operator is selected, there is always $P_{\Omega}(X(t)) \neq 0$; in other words, $P_{\Omega}(X(t)) \notin \operatorname{Null}\left(P_{\Omega}\right)$, which meets the Null Space Property.
3.2. The FPC-ROOT Algorithm. Suppose $\operatorname{svd}\left(X_{R}(t)\right)=U \Sigma V$ and matrices $U$ and $V$ are the left and right singular value matrix of $X_{R}(t)$. FPC-ROOT algorithm can obtain $U$ and $V$ directly by matrix completion based on the low-rank property of $X(t)$ and realizes 2D DOA estimation by computing the polynomial roots.

Firstly, to solve problem (12), we achieve the left and right singular value matrices $U$ and $V$ of estimated value $X_{R}(t)$ in full array by FPC algorithm. And then, we solve the autocorrelation matrix of received signal $X(t)$ in full array, so the eigendecomposition of the autocorrelation matrix is

$$
\begin{aligned}
R_{X} & =X(t) X(t)^{H}=A_{x} S(t) A_{y}^{T} A_{y}^{*} S(t)^{*} A_{x}^{H}+N_{m 1} \\
& =A_{x} R_{m 1} A_{x}^{H}+N_{m 1}=U_{S} \Sigma_{S} U_{S}^{H}+U_{N} \Sigma_{N} U_{N}^{H},
\end{aligned}
$$

where $t=1, \ldots, W, X(t)^{H}$ is the conjugate transpose matrix of $X(t), U_{S}$ is the signal subspace, $U_{N}$ is the noise subspace, and $R_{m 1}$ is

$$
R_{m 1}=S(t) A_{y}^{T} A_{y}^{*} S(t)^{*} .
$$

And $N_{m 1}$ in (15) can be expressed as

$$
\begin{aligned}
N_{m 1}= & A_{x} S(t) A_{y}^{T} N_{R}(t)^{H}+N_{R}(t) A_{y}^{*} S(t)^{*} A_{x}^{H} \\
& +N_{R}(t) N_{R}(t)^{H} .
\end{aligned}
$$

It is obvious that the spanned subspaces of $A_{x}$ and $U_{S}$ are identical. We solve the conjugate transpose of autocorrelation matrix $R_{X}$ and then it can be concluded that

$$
\begin{aligned}
R_{X}^{H} & =X(t)^{H} X(t)=A_{y}^{*} S(t)^{*} A_{x}^{H} A_{x} S(t) A_{y}^{T}+N_{m 2} \\
& =A_{y}^{*} R_{m 2} A_{y}^{T}+N_{m 2}=V_{S} \Sigma_{S} V_{S}^{H}+V_{N} \Sigma_{N} V_{N}^{H},
\end{aligned}
$$

where $t=1, \ldots, N$, and similarly $R_{m 2}$ is

$$
R_{m 2}=S(t)^{*} A_{x}^{H} A_{x} S(t)
$$

$N_{m 2}$ can be expressed as

$$
\begin{aligned}
N_{m 2}= & A_{y}^{*} S(t)^{*} A_{x}^{H} N_{R}(t)+N_{R}(t)^{H} A_{x} S(t) A_{y}^{T} \\
& +N_{R}(t)^{H} N_{R}(t) .
\end{aligned}
$$

So the space spanned by $A_{y}^{*}$ is the same as the space spanned by $V_{S}$.

Based on singular value decomposition, it is easy to know that left and right singular value matrices $U$ and $V$ are related to the eigendecomposition of autocorrelation matrix $R_{X}$ by

$$
\begin{aligned}
& U=U_{S}+U_{N}, \\
& V=V_{S}+V_{N} .
\end{aligned}
$$

So the left and right singular vectors of received signal matrix can be obtained directly from the output of matrix completion algorithm instead of eigendecomposing autocorrelation matrix of received signal and the computational complexity of the proposed algorithm obtained a corresponding reduction. 
Consider a polynomial as

$$
f(z)=u_{l}^{H} p(z) \quad(l=k+1, k+2, \ldots, N),
$$

where $u_{l}$ is the $l$ th eigenvector of matrix $R_{X}$ and $p(z)=$ $\left[1, z, \ldots, z^{N-1}\right]^{T}$. It can be deduced that the corresponding ROOT-MUSIC polynomial is

$$
f_{x}(z)=z^{N-1} p^{T}\left(z^{-1}\right) U_{N} U_{N}^{H} p(z) .
$$

By solving the polynomial roots, the proposed algorithm can avoid scanning of the $2 \mathrm{D}$ spectral peak. Taking use of the orthogonality of signal subspace, the above equation can be converted to

$$
P_{x}(z)=z^{N-1} p^{T}\left(z^{-1}\right)\left(I-U_{S} U_{S}^{H}\right) p(z) .
$$

The dimensionality of $U_{S}$ is smaller than $U_{N}$; hence, the proposed algorithm effectively reduced the computational complexity by reducing the dimension of the subspace matrix. Then, the vector corresponding to the roots of polynomial is

$$
\begin{gathered}
z_{x}=\left[\exp \left(\frac{j 2 \pi d_{x} \cos \phi_{1} \sin \theta_{1}}{\lambda}\right), \ldots,\right. \\
\left.\quad \exp \left(\frac{j 2 \pi d_{x} \cos \phi_{k} \sin \theta_{k}}{\lambda}\right)\right] .
\end{gathered}
$$

Thus,

$$
r_{x}=\left[\cos \phi_{1} \sin \theta_{1}, \ldots, \cos \phi_{k} \sin \theta_{k}\right] .
$$

Similarly, it can be obtained that

$$
r_{y}=\left[\sin \phi_{1} \sin \theta_{1}, \ldots, \sin \phi_{k} \sin \theta_{k}\right] .
$$

Hence, the estimated angles of targets are

$$
\begin{aligned}
& {\left[\theta_{1}, \theta_{2}, \ldots, \theta_{k}\right]=\arcsin \sqrt{r_{x}^{2}+r_{y}^{2}}} \\
& {\left[\phi_{1}, \phi_{2}, \ldots, \phi_{k}\right]=\arctan \left(\frac{r_{x}}{r_{y}}\right) .}
\end{aligned}
$$

In summary, the proposed FPC-ROOT algorithm in this paper can be programmed as follows.

\section{Fixed Point Continuation Polynomial Roots (FPC-ROOT) Algorithm}

Initialize. Given $X_{s}(t)$, the projection $P_{\Omega}$ is corresponding to element positions in the sparse array:

(1) Solve (12) to get left and right singular value matrices $U_{S}$ and $V_{S}$.

(2) Construct the polynomial roots (24).

(3) Solve (24) to get

$$
\begin{aligned}
& r_{x}=\left[\cos \phi_{1} \sin \theta_{1}, \ldots, \cos \phi_{k} \sin \theta_{k}\right], \\
& r_{y}=\left[\sin \phi_{1} \sin \theta_{1}, \ldots, \sin \phi_{k} \sin \theta_{k}\right] .
\end{aligned}
$$

(4) Determine the targets angles

$$
\begin{aligned}
& {\left[\theta_{1}, \theta_{2}, \ldots, \theta_{k}\right]=\arcsin \sqrt{r_{x}^{2}+r_{y}^{2}},} \\
& {\left[\phi_{1}, \phi_{2}, \ldots, \phi_{k}\right]=\arctan \left(\frac{r_{x}}{r_{y}}\right) .}
\end{aligned}
$$

End.

3.3. Dimensionality Reduction. In normal 2D-DOA estimation, DOA estimation is achieved through autocorrelation matrix of a vector which is transformed from the signal matrix. Suppose the signal matrix is $M_{x} \times M_{y}$, so the dimension of the transformed vector is $M_{x} M_{y} \times 1$; then, the dimension of the autocorrelation matrix is $M_{x} M_{y} \times M_{x} M_{y}$. In the proposed method, we obtain left and right singular vector directly from the signal matrix, avoiding solving the autocorrelation matrix, greatly reducing the dimension of the target matrix.

\section{Simulations}

In this section, several simulations for 2D DOA estimation are conducted to demonstrate the feasibility and effectiveness of the proposed algorithm in sparse array. In these experiments, we sample 1200 elements from full array at random to formulate a sparse array. The full array is an URA of $64 \times 64$ elements, where the total number of elements is $m=4096$ and the corresponding element spacing in $x$-direction and $y$ direction is $d_{x}=d_{y}=\lambda / 2$.

In the first examples, 2D DOA estimation of sparse array is shown. Let three targets be in the space domain, whose elevation and azimuth angles of $2 \mathrm{D}$-DOA are $\left(10^{\circ}, 15^{\circ}\right)$, $\left(20^{\circ}, 25^{\circ}\right)$, and $\left(30^{\circ}, 35^{\circ}\right)$, respectively, and input signal-tonoise ratio (SNR) is $20 \mathrm{~dB}$. Let the number of snapshots be 50. Suppose the received signals are narrow-band in far field and the signal sources are uncorrelated; then, 100 experiment results of 2D-DOA estimation by FPC-ROOT algorithm are depicted in Figure 2. The proposed algorithm samples 1200 elements to construct a sparse array; in other words, $70 \%$ units are removed from full array. From Figure 2, it can be seen that the targets' angles can be achieved precisely by the proposed algorithm in sparse array.

In the second experiment, recovery errors by matrix completion in sparse array with different SNR are examined. We recover a full matrix from a sparse matrix using FPC algorithm. Suppose the full matrix is $M$ and the recovered matrix is $X$; then, the measurement criterion of recovery errors by matrix completion is $\|M-X\|_{2} /\|M\|_{2}$. Figure 3 illustrates the variation of recovery errors by matrix completion with different SNR when the numbers of sparse array elements are 900, 1200, and 1500, respectively. It can be deduced easily from this experiment that recovery errors by FPC algorithm are inversely proportional to SNR. The larger the SNR is, the more similar the matrix $M$ is to low-rank matrix. Simultaneously, the more elements the sparse array has, the closer the recovered signals are to the full array and the smaller the corresponding recovery errors are. 


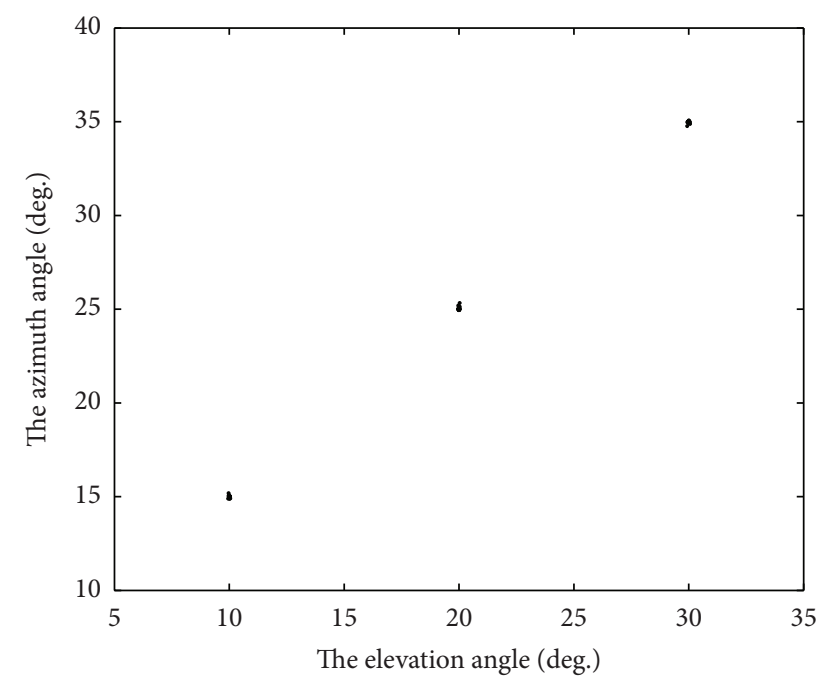

FIGURE 2: 2D DOA estimation via FPC-ROOT algorithm.

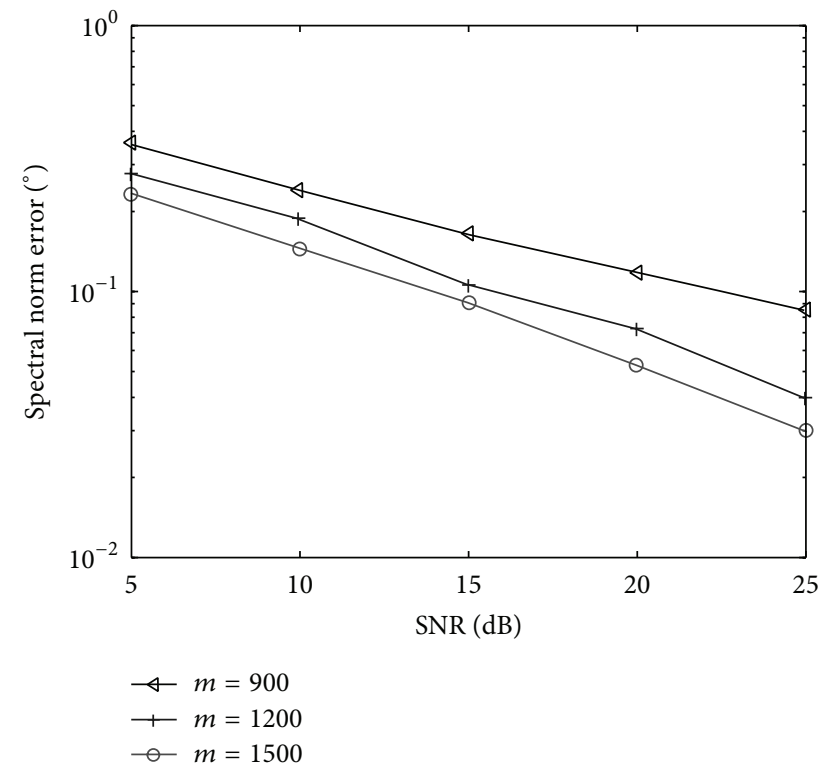

FIGURE 3: Recovery errors by matrix completion with different elements and SNR.

In the third experiment, root mean square error (RMSE) of $2 \mathrm{D}$ DOA estimation based on matrix completion is discussed. When 500 Monte Carlo simulations are performed, Figure 4 exhibits RMSE with different SNR via various algorithms. When the SNR is high, the recovery of matrix completion has a high precision, so the RMSE of DOA estimation is decreased. And it is evident that the proposed algorithm has higher accuracy for both elevation and azimuth angles estimation than 2D-MUSIC algorithms.

In the last experiment, RMSE by FPC-ROOT algorithm with different SNR and elements is demonstrated. Let the number of snapshots be 50 and let 500 Monte Carlo experiments be implemented. Figure 5 shows RMSE by FPC-ROOT

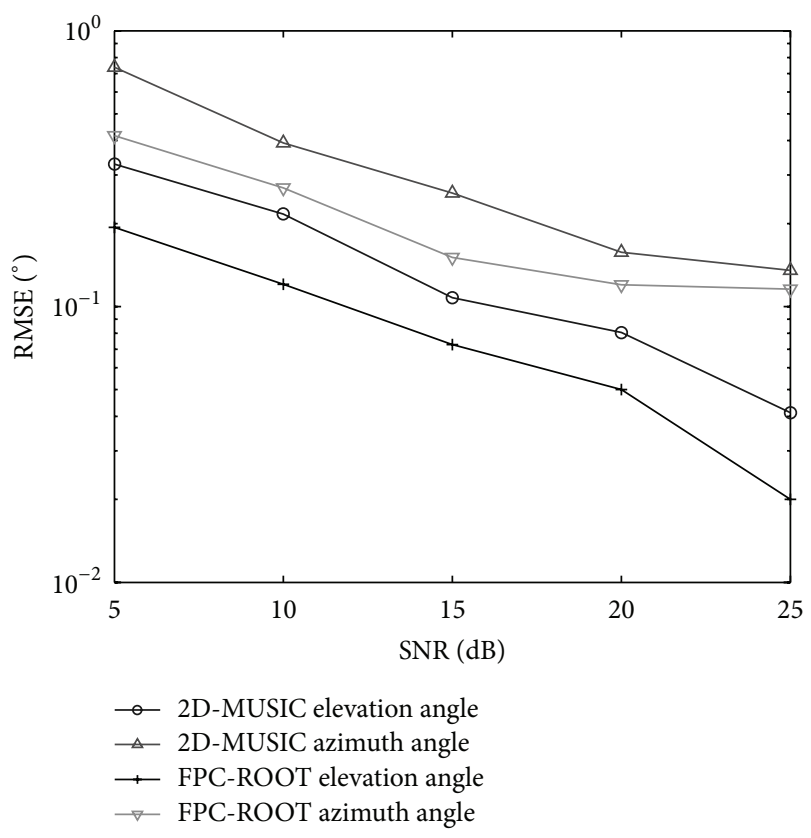

FIGURE 4: RMSE of 2D DOA estimation based on matrix completion.

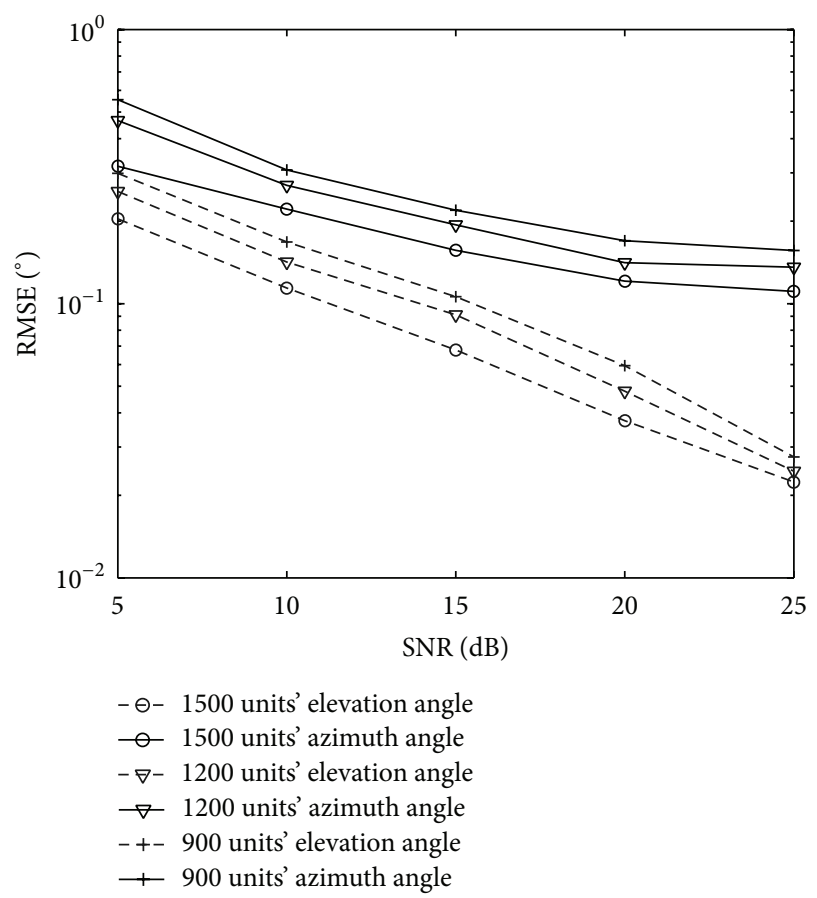

FIGURE 5: RMSE versus SNR with different elements by FPC-ROOT algorithm.

algorithm when SNR and the number of elements vary. As the recovery errors by FPC algorithm are inversely proportional to SNR, the RMSE of DOA estimation is decreased at high SNR. From this simulation, it can be concluded that the estimation accuracy by FPC-ROOT algorithm is proportional to the number of elements. 


\section{Conclusion}

In this paper, a FPC-ROOT algorithm is proposed based on matrix completion, which can achieve high accuracy of $2 \mathrm{D}$ DOA estimation with reduced antenna units. The proposed algorithm obtains left and right singular vectors of received signal by the output of matrix completion algorithm directly instead of eigendecomposing the autocorrelation matrix of received signal, for the benefit of lower dimensions. Besides, by computing polynomial roots, the proposed algorithm can avoid the scanning of two-dimensional spectral peak, which cuts down the computational complexity.

\section{Competing Interests}

The authors declare that there is no conflict of interests regarding the publication of this paper.

\section{Acknowledgments}

This work was supported by the National Natural Science Foundation of China (Grant no. 61401204), Postdoctoral Science Foundation of Jiangsu Province (Grant no. 1501104C), Technology Research and Development Program of Jiangsu Province (Grant no. BY2015004-03), and the Fundamental Research Funds for the Central Universities (Grant no. 30916011319).

\section{References}

[1] J. Li and X. Zhang, "Closed-form blind 2D-DOD and 2D-DOA estimation for MIMO radar with arbitrary arrays," Wireless Personal Communications, vol. 69, no. 1, pp. 175-186, 2013.

[2] Z. Zheng, G. Li, and Y. Teng, "Simplified estimation of 2D DOA for coherently distributed sources," Wireless Personal Communications, vol. 62, no. 4, pp. 907-922, 2012.

[3] P. Heidenreich, A. M. Zoubir, and M. Rubsamen, "Joint 2-D DOA estimation and phase calibration for uniform rectangular arrays," IEEE Transactions on Signal Processing, vol. 60, no. 9, pp. 4683-4693, 2012.

[4] M. Agatonović, Z. Stanković, N. Dončov, L. Sit, B. Milovanović, and T. Zwick, "Application of artificial neural networks for efficient high-resolution 2D DOA estimation," Radioengineering, vol. 21, no. 4, pp. 1178-1186, 2012.

[5] W. Zhang, W. Liu, J. Wang, and S. Wu, "Computationally efficient 2-D DOA estimation for uniform rectangular arrays," Multidimensional Systems and Signal Processing, vol. 25, no. 4, pp. 847-857, 2014.

[6] L. Yuan, R. Jiang, and Y. Chen, "Gain and phase autocalibration of large uniform rectangular arrays for underwater 3-D sonar imaging systems," IEEE Journal of Oceanic Engineering, vol. 39, no. 3, pp. 458-471, 2014.

[7] N. Hu, Z. Ye, X. Xu, and M. Bao, "DOA estimation for sparse array via sparse signal reconstruction," IEEE Transactions on Aerospace \& Electronic Systems, vol. 49, no. 2, pp. 760-773, 2013.

[8] O. Klopp, "Noisy low-rank matrix completion with general sampling distribution," Bernoulli, vol. 20, no. 1, pp. 282-303, 2014.

[9] H. Ji, C. Liu, Z. Shen, and Y. Xu, "Robust video denoising using low rank matrix completion," in Proceedings of the IEEE
Conference on Computer Vision and Pattern Recognition (CVPR '10), pp. 1791-1798, San Francisco, Calif, USA, June 2010.

[10] E. J. Candès and B. Recht, "Exact matrix completion via convex optimization," Foundations of Computational Mathematics, vol. 9, no. 6, pp. 717-772, 2009.

[11] D. L. Donoho, "Compressed sensing," IEEE Transactions on Information Theory, vol. 52, no. 4, pp. 1289-1306, 2006.

[12] J. Cheng, Q. Ye, H. Jiang, D. Wang, and C. Wang, "STCDG: an efficient data gathering algorithm based on matrix completion for wireless sensor networks," IEEE Transactions on Wireless Communications, vol. 12, no. 2, pp. 850-861, 2013.

[13] W. Chen, Q. Tian, J. Liu, and Q. Wang, "Nonlocal low-rank matrix completion for image interpolation using edge detection and neural network," Signal, Image and Video Processing, vol. 8, no. 4, pp. 657-663, 2014.

[14] D. S. Kalogerias and A. P. Petropulu, "Matrix completion in colocated mimo radar: recoverability, bounds \& theoretical guarantees," IEEE Transactions on Signal Processing, vol. 62, no. 2, pp. 309-321, 2014.

[15] S. Ma, D. Goldfarb, and L. Chen, "Fixed point and Bregman iterative methods for matrix rank minimization," Mathematical Programming, vol. 128, no. 1-2, pp. 321-353, 2011.

[16] J. Tanner and K. Wei, "Normalized iterative hard thresholding for matrix completion," SIAM Journal on Scientific Computing, vol. 35, no. 5, pp. S104-S125, 2013.

[17] B. Recht, W. Xu, and B. Hassibi, "Necessary and sufficient conditions for success of the nuclear norm heuristic for rank minimization," in Proceedings of the 47th IEEE Conference on Decision and Control (CDC '08), pp. 3065-3070, Cancún, Mexico, December 2008.

[18] P. Pal and P. P. Vaidyanathan, "A grid-less approach to underdetermined direction of arrival estimation via low rank matrix denoising," IEEE Signal Processing Letters, vol. 21, no. 6, pp. 737741,2014

[19] H. Krim and M. Viberg, "Two decades of array signal processing research: the parametric approach," IEEE Signal Processing Magazine, vol. 13, no. 4, pp. 67-94, 1996. 


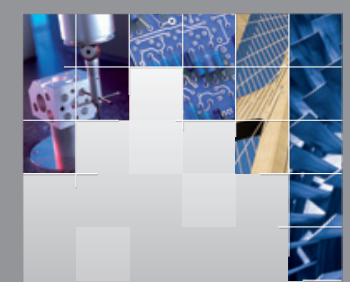

\section{Enfincering}
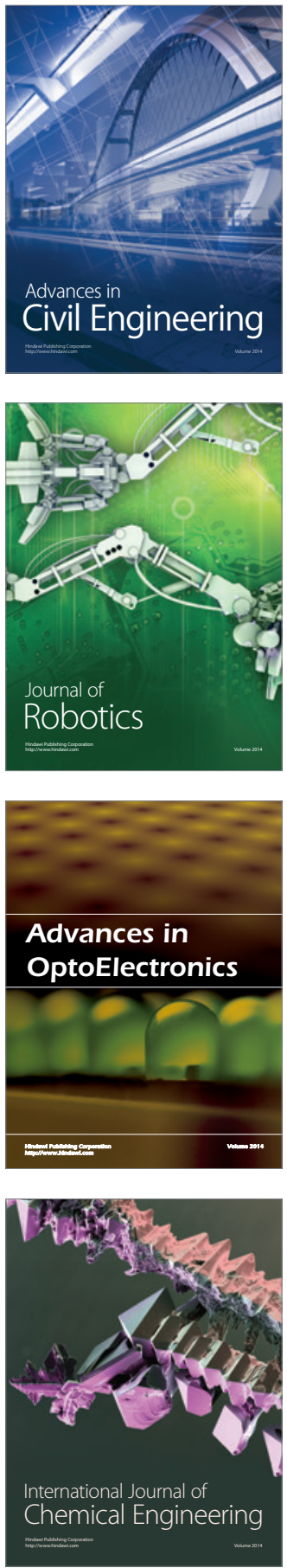

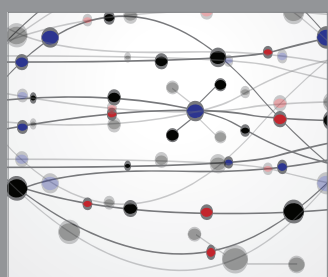

The Scientific World Journal

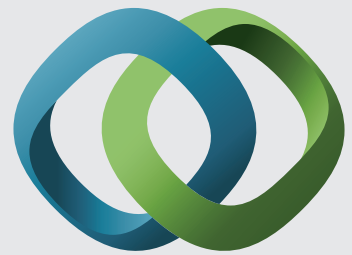

\section{Hindawi}

Submit your manuscripts at

http://www.hindawi.com
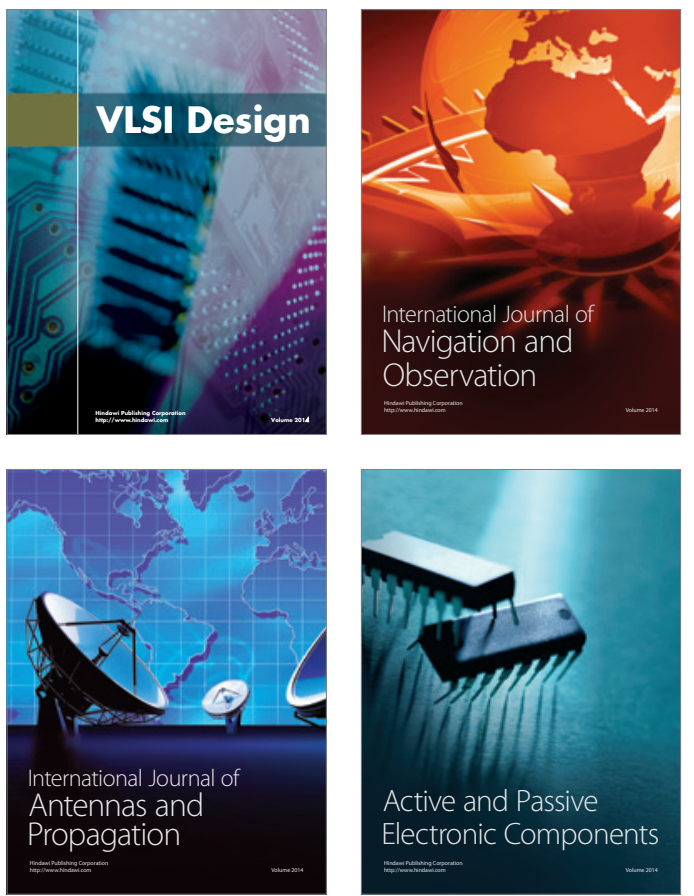
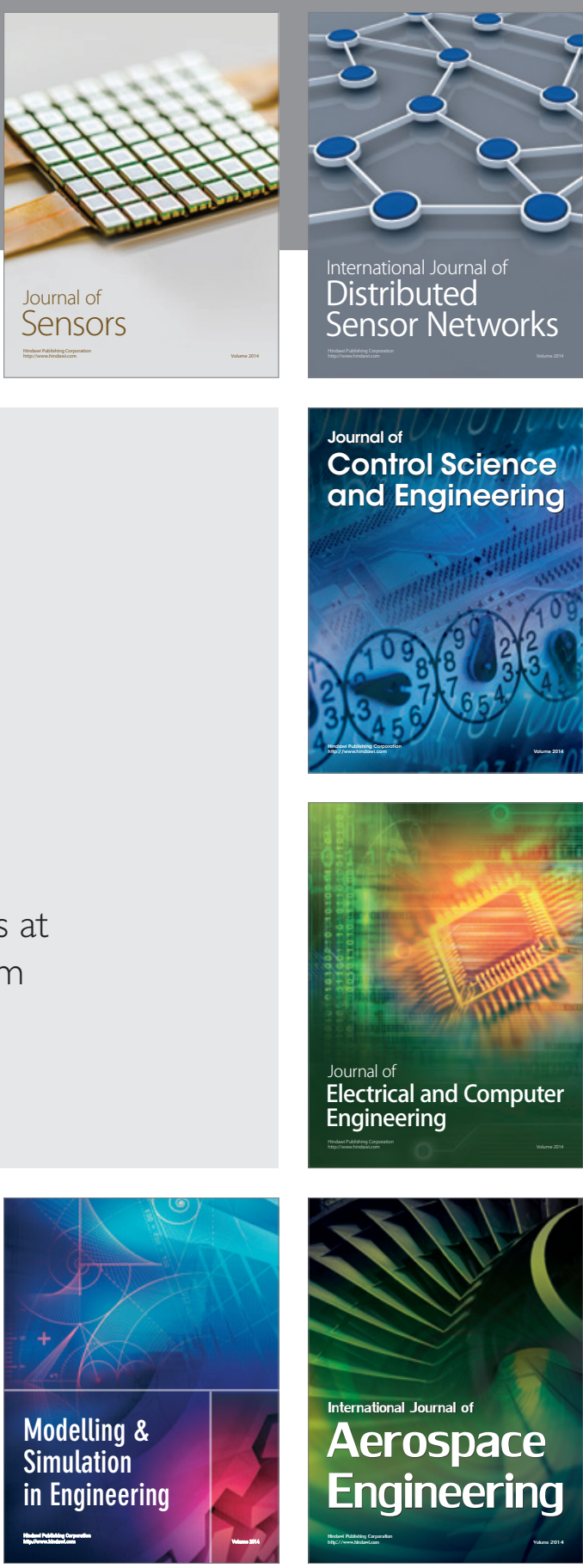

International Journal of

Distributed

Sensor Networks

Journal of

Control Science

and Engineering
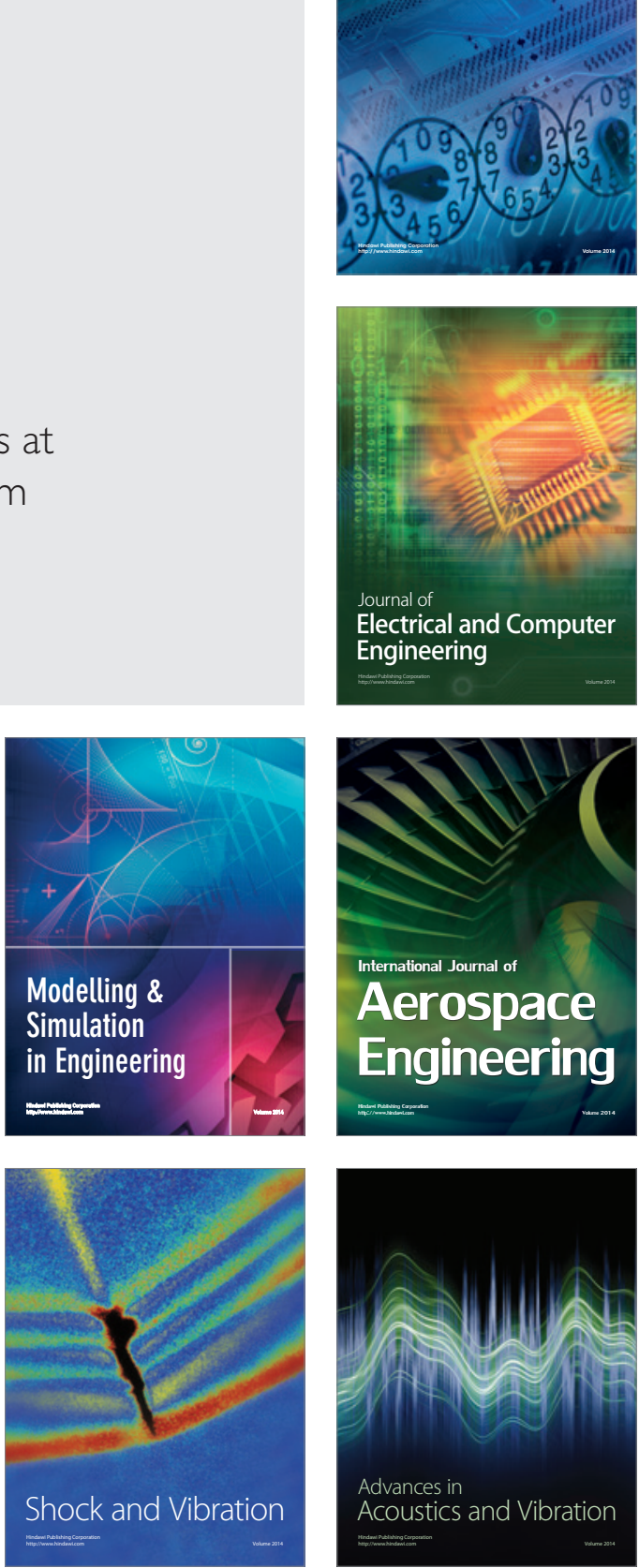